\title{
Клиническая медицина
}

УДК 616.345

\section{ДИВЕРТИКУЛЯРНАЯ БОЛЕЗНЬ ТОЛСТОГО КИШЕЧНИКА: СОВРЕМЕННОЕ СОСТОЯНИЕ ПРОБЛЕМЫ}

\author{
1Лысов Н.А., 1, 2Осадчук М.М., Балашов Д.В. \\ 1Частное учреждение образовательная организация высшего образования \\ «Медицинский университет Реавиз», Самара \\ 2ГБУЗ г. Москвы «Городская поликлиника № 52 Департамента здравоохранения города Москвы», \\ Москва
}

\begin{abstract}
Резюме. В данном обзоре приведены современные сведения об этиопатогенезе, клинике, диагностике и лечении дивертикулярной болезни толстого кишечника (ДБТК). На основании проведенного анализа и эпидемиологических данных данную патологию можно отнести к глобальной эпидемии 21 века, ассоциированной с вестернизацией образа жизни и постарением населения в цивилизованных странах. Описанная тактика ведения больных с данной патологией базируется на мировых тенденциях, сформировавшихся в последнее десятилетие.
\end{abstract}

Конфликт интересов. Авторы заявляют об отсутствии конфликта интересов.

Финансирование. Исследование проводилось без спонсорской поддержки.

Ключевые слова: дивертикулярная болезнь толстой кишки, дивертикулит, рифаксимин, месалазин.

Для цитирования: Лысов Н.А., Осадчук М.М., Балашов Д.В. Дивертикулярная болезнь толстого кишечника: современное состояние проблемы // Вестник медицинского института «Реавиз». - 2020. - № 4. C. $33-41$.

\section{DIVERTICULAR DISEASE OF THE LARGE INTESTINE: CURRENT PROBLEM STATE}

\author{
${ }^{1}$ Lysov N.A., ${ }^{1,2}$ Osadchuk M.M., ${ }^{2}$ Balashov D.V.
}

${ }^{1}$ Private institution educational organization of higher education

"Medical University Reavies, Samara

${ }^{2}$ GBUZ of Moscow «City Polyclinic № 52 of the Moscow Department of Health», Moscow

\begin{abstract}
This review presents modern information on etiopathogenesis, clinic, diagnostics and treatment of diverticular disease of the large intestine (DBTK). Based on the analysis and epidemiological data, this pathology can be attributed to the global epidemic of the 21st century, associated with the Westernization of lifestyle and aging of the population in civilized countries. The described tactics of management of patients with this pathology is based on global trends that have formed in the last decade.
\end{abstract}


Conflict of interest. The authors declare that there is no conflict of interest.

Financing. The research was conducted without sponsorship.

Key words: diverticular disease of the colon, diverticulitis, rifaximine, mesalazine.

To cite: Lysov N.A., Osadchuk M.M., Balashov D.V. Diverticular disease of the large intestine: current state of the problem // Bulletin of the Medical Institute "Reaviz". - 2020. - № 4. - P. 33-41.

Дивертикулярная болезнь толстой кишки (ДБТК) является глобальной проблемой современного цивилизованного мира. Так, ДБТК входит в шестерку наиболее распространенных заболеваний желудочно-кишечного тракта у амбулаторных больных и одной из основных причин госпитализации в США с оценочной стоимостью 5,5 миллиарда долларов [1, 2]. Национальные данные рутинной клинической колоноскопии в США свидетельствуют о том, что распространенность дивертикулеза составляет 20 \% среди лиц в возрасте 40-49 лет и достигает 75 \% у лиц 80 лет и старше. $[3,4]$. При этом при колоноскопическом исследовании, направленном на выявление дивертикулеза, плотность, размер, глубина и распределение дивертикулов также увеличивается с возрастом. Необходимо отметить, что примерно у 10-25 \% людей с дивертикулезом кишечника заболевание осложняется дивертикулитом, который относится к наиболее частым показаниям для проведения колэктомии [5, 6]. Заслуживают внимание статистические данные, свидетельствующие о том, что рост оперативных вмешательств при дивертикулитах увеличился до 29 \%, особенно у молодых людей (73 \% для этих лиц) [7]. При этом в Европе на ДБТК приходится около 13000 смертей в год [8].

Таким образом, в странах Западной Европы и США из-за высокой распространенности ДБТК, особенно в пожилом возрасте, она относится к 5-6 наиболее важным желудочно-кишечным заболеваниям как с точки зрения прямых, так и косвенных расходов со смертностью 2,5 на 100000 населения в год [9]. Особую тревогу вызывает тот факт, что ДБТК является значительной проблемой не только для нацио- нальных систем здравоохранения развитых стран, но становится заметной проблемой и в странах, где она до недавнего времени была менее актуальной [10]. Полагают, что рост заболеваемости ДБТК связан с вестернизацией национального здравоохранения, что сопровождается значительными экономическими затратами [11].

ДБТК и колоректальный рак (КР) носят ассоциированный характер за счет наличия одних и тех же провоцирующих факторов: пожилой возраст, дефицит пищевых волокон при абсолютном доминировании животных жиров, переход на употребление субпродуктов. Это находит подтверждение и в клинической практике. Так, при колоноскопии у 30 \% больных с ДБТК верифицируются полипы толстой кишки и у 9 \% с данной патологией - колоректальный рак (КР) [12]. Вместе с тем отсутствуют убедительные данные о тесной патогенетической связи ДБТК с полипами кишечника и КР изза незначительных различий в распространенности неоплазий в одной возрастной группе лиц с ДБТК и без нее [2, 13]. В связи с этим важное значение приобретает изучение регуляторов канцерогенеза и пролиферации для оценки общих и частных механизмов возникновения и прогрессирования данной патологии у лиц с ДБТК и при ее ассоциации с полипами кишечника и КР.

Этиопатогенез ДБТК остается неясной, клинические проявления широко варьируют и прогноз заболевания трудно прогнозируем. Существенную потенциальную роль в развитии ДБТК принадлежит дисфункции соединительной ткани, которая лучше всего иллюстрируется ассоциацией дивертикулеза с наследственными заболеваниями соединительной ткани, в том числе Марфана, Элерса-Данло, Coffin-Lowry, и 
Williams синдромом и старением. Наиболее часто встречающимися факторами риска развития ДБТК выступают нестероидные противовоспалительные препараты [14]. В качестве факторов риска указывают на ишемическую болезнь сердца, артериальную гипертензию и ожирение [15]. Важное значение в формировании ДБТК, и особенно тяжести ее течения, придают микробиоте, играющей определяющую роль в патогенезе дивертикулита. [16, 17]. Так, дисбактериоз кишечника связан с дефектами слизистой оболочки и локальной иммунной дисфункцией, и эти нарушения могут способствовать воспалению, ведущему к развитию дивертикулита. Использование антибиотиков также приводит к формированию несбалансированного микробного сообщества и манифестации минимального воспаления, что связано с риском развития ДБТК и дивертикулита [18]. Генетические исследования показали семейную ассоциацию со специфическим геном TNFSF в прогнозирования тяжести заболевания [19].

Несмотря на огромную распространенность во всем мире ДБТК и ее социальноэкономическое воздействие на современное общество, патогенез данного заболевания изучен достаточно поверхностно, и большинство литературных данных относится к 1960/70/80-х годам прошлого столетия. Так, литературных источников, изучающих и анализирующих патогенез воспалительных заболеваний кишечника, артериальной гипертензии и сахарного диабета в 60 раз больше, по сравнению с аналогичными работами по ДБТК толстой кишки [20].

Морфологическим субстратом ДБТК являются псевдокисты, манифестирующие с возрастом. Их форма напоминает «мешочки», и при исследовании данные образования представляют собой подслизистую грыжу, проникающую через сосудистые порталы в собственную мышечную ткань в субсерозу.
Большое значение в определении тактики ведения больных с ДБТК принадлежит классификационным критериям. Наиболее популярной является эндоскопическая классификация ДБТК. Это тем более удивительно, если учесть, что наибольшее количество больных с ДБТК в последние два десятилетия выявляются именно при колоноскопии [21] и при этом эндоскопические признаки дивертикулярного воспаления при первичном обследовании находят уже у 0,48-1,7 \% пациентов [22]. Кроме того, некоторые эндоскопические характеристики толстой кишки при ДБТК нередко определяют прогноз заболевания, а в сочетании с радиологическими исследованием выступают как один из наиболее значимых предикторов рецидивирующего дивертикулита [22, 23].

Оптимальным классификационным критериям соответствует систематизация DICA, которая состоит из четырех эндоскопических элементов [24, 25], (табл. 1):

1. Расширение дивертикула левого или правого.

2. Количество дивертикулов (в каждом отделе): до 15 (класс I), более чем 15 (класс II).

3. Наличие воспаления: отек/гиперемия; эрозии; сегментарный колит, связанный с дивертикулезом.

4. Осложнения: жесткость толстой кишки - плохое растяжение дивертикулярной области кишки, также включая незначительный стеноз, позволяющий пройти этот участок стандартным колоноскопом; стеноз, не дающий возможность пройти его стандартным колоноскопом, в том числе из-за повышенного риска перфорации, наличия некоторых анатомических характеристик (например, несколько дивертикулов на селезеночном изгибе); наличие абсцесса, микроперфорации; кровотечения. 
Таблица 1. Оценка тяжести дивертикулярной болезни (DICA)

\begin{tabular}{|l|c|}
\hline \multicolumn{1}{|c|}{ Элементы } & Баллы \\
\hline $\begin{array}{l}\text { Расширение дивертикула } \\
\text { левой части кишки } \\
\text { правой части кишки }\end{array}$ & 2 \\
\hline Количество дивертикулов в каждом отделетолстой кишки \\
до 15: 1-й класс \\
> 15: ॥-й класс & 0 \\
\hline Эндоскопические признаки воспаления & 1 \\
отек/гиперемия & 1 \\
эрозии & 2 \\
сегментарный колит, ассоциированный с дивертикулезом & 3 \\
\hline Настоящие осложнения & 4 \\
«Жесткая» кишка» & 4 \\
Стеноз & 4 \\
Гнойник (абсцесс) & 4 \\
Кровотечение & \\
\hline Для полного описания и объяснения Этой классификации, пожалуйста, прочитайте \\
текст
\end{tabular}

Примечание: DICA I от 1 до 3 баллов; DICA II от 4 до 7 баллов; DICA III > 7 баллов.

Таким образом, DICA был классифицирован как DICA I (до трех баллов); DICAll (4-7 баллов); DICA III (более 7 баллов).

Оценка тяжести течения ДБTK по DICA, ассоциирована со следующими показателями: боль в животе (длительность более месяца), повышение скорости оседания эритроцитов (СОЭ), положительный тест на фекальный кальпротектин. Последний выступает в качестве надежного и недорогого показателя, способного помочь врачу в диагностике, оценке активности и мониторинге острого дивертикулита. Еще важнее то, что он коррелирует с ответом на терапию и может прогнозировать развитие рецидива дивертикулита. Фекальный кальпротектин выступает также надежным индикатором выявления субклинического воспаления кишечника, что позволяет с высокой степенью достоверности разграничить данную патологию с синдромом раздраженного кишечника (СРК).

На основании многочисленных данных, полученных из источника medline, определение С-реактивного белка (СРБ) дает возможность ранней верификации дивертикулита. Так, при его значениях > 50 мг/л. он в состоянии конкурировать с другими сывороточными биомаркерами в диагностике данного осложнения. Кроме этого, СРБ обладает способностью высокой степени корреляции с гистологической картиной и тяжестью воспаления при остром дивертикулите, риске перфорации, и в оценке ответа на проводимую терапию [26].

Таким образом, DICA I соотносится с простым дивертикулезом, DICA II может варьировать между тяжелым дивертикулезом и мягким дивертикулитомом, DICA III может рассматриваться как более тяжелый дивертикулит или как осложнение дивертикулита (то есть стеноз).

Спектр дивертикулярной болезни широк и охватывает различные клинические сценарии, каждый из которых характеризуется различной симптоматикой, степенью тяжести и исходами. Патогенез клинических симптомов, а также связь между неосложненным дивертикулезом и симптомами сложны и до сих пор не до конца понятны. Однако слабое воспаление, вызванное бактериальной иммунной активацией, определяет патофизиологическую основу симптоматики дивертикулеза кишечника

Клиника ДБТК может быть острой или хронической Хронические проявления заболевания сходны с симптомами синдрома раздраженного кишечника [СРК] (например, 
боль в животе и дискомфорт, вздутие живота, запоры и диарея). Острые симптомы ДБТК могут напоминать аппендицит (например, лихорадка, острые боли в животе и лейкоцитоз) [27].

Кишечная симптоматика дивертикулеза в основном соответствует клинике СРК. Поэтому при наличии данной симптоматики у лиц старше 50 лет необходимо исключать наличие ДБТК. Подобная практика находит подтверждение и в рекомендациях Общества колоректальных хирургов США (2000), Эти рекомендации подчеркивают, что для ДБТК характерен комплекс клинических признаков, ассоциированных с болевым синдромом разной степени интенсивности, вздутием живота и нарушением стула.

Первую группу больных с ДБТК составляют больные с отсутствием кишечной симптоматики, дивертикулы обнаруживают у них при поиске других заболеваний. У 70 \% больных ДБТК остается бессимптомной на протяжении всей жизни. Вторая группа больных представлена лицами с клинически выраженными проявлениями дивертикулеза. Однако характерных симптомов неосложненного дивертикулеза нет. У больных наблюдаются признаки, типичные для СРК, - боли в животе без четкой локализации, нарушения стула, чаще всего запоры, признаки кишечной диспепсии (урчание, вздутия). Кроме того, больные нередко жалуются на чувство неполного опорожнения кишки и вздутие живота. У части больных наблюдается чередование запора и появления жидкого стула.

К осложнениям ДБТК следует относить дивертикулит, перфорацию, кровотечение, стеноз, кишечную непроходимость, абсцесс, свищ и сенгментарный колит. В клинике выделяют острый неосложненный и осложненный дивертикулит. Особое внимание в последние годы уделяется вялотекущему дивертикулиту, симптоматика которого не соответствует клинике быстро разрешающегося дивертикулита. Для него характерно длительное течение, укладывающееся в вялотекущий неосложненный и атипичный дивертикулит. У небольшой части пациентов с ДБТК может развиваться сегментарный колит, связанный с дивертикулитом [28]. Сегментарный колит ассоциируется с конкретными макроскопическими и микроскопическими параметрамии, характеризуется хроническим, иногда рецидивирующим течением, воспалением слизистой оболочки в зоне наличия дивертикулов, обычно В области сигмовиднойнисходящей части ободочной кишки, не затрагивая проксимальный отдел данного отдела пищеварительного тракта и прямую кишку. Дивертикулярное кровотечение наблюдается преимущественно у пожилых пациентов, с максимальной распространенностью в девятом десятилетии жизни. Примерно у 10 \% пациентов он может носить рецидивирующий характер.

ДБТК является распространенным заболеванием, однако его эффективное лечение является сложной задачей в клинической практике. Диагноз дивертикулярной болезни может быть легким, [29] его ведение является трудоемким, поскольку рандомизированные контролируемые испытания по лечению при различных вариантах течения являются скудными, часто носящие разноплановый характер и имеют низкое качество или отрицательные результаты [30]. Ведение этих пациентов часто следует традиции или мнению экспертов, а не доказательной медицине. Аналогичным образом, существующие практические рекомендации основаны главным образом на мнении экспертов [30, 31]. Большинство существующих методов ведения больных с данной патологией не опираются на международные и национальные руководящие принципы в зависимости от уровня доказательств [32, 33].

Цели терапии симптоматической неосложненной ДБТК: сначала отличить ДБТК от СРК; затем купировать симптомы заболевания и улучшить качество жизни и предотвратить прогрессирование до острого дивертикулита. 
Цели терапии у пациентов, у которых развивается острый дивертикулит, включают купирование симптомов, ликвидацию кишечной инфекции, предотвращение осложнений и рецидива заболевания [34] (табл. 2).

Таблица 2. Цели терапии больных с острым дивертикулитом

\begin{tabular}{|c|c|}
\hline $\begin{array}{c}\text { Направление медицинской } \\
\text { деятельности }\end{array}$ & Тактика \\
\hline Просилактика & $\begin{array}{l}\text { 1. Нивелирование управляемых факторов риска. } \\
\text { 2. Поддержание ремиссии у больных с ДБТК }\end{array}$ \\
\hline Лечение & $\begin{array}{l}\text { 1. Сокращение ненужных госпитализаций. } \\
\text { 2. Сокращение нецелевого использования противомикробных } \\
\text { препаратов. } \\
\text { 3. Сокращение продолжительности пребывания в стационар } \\
\text { 4. Уменьшение и вероятное предотвращение осложнений. }\end{array}$ \\
\hline Профилактика рецидивов & Надлежащее управление факторами риска рецидива \\
\hline
\end{tabular}

Основными классами фармакологической терапии, которые в настоящее время применяются при ДБТК, являются противовоспалительные препараты (месалазин) и плохо всасываемые антибиотики (а именно рифаксимин). Обоснование использования аминосалицилатов, таких как месалазин, основано на предположении о слабом воспалении при ДБТК и возникновении симптомов, тогда как явное воспаление может вызывать дивертикулит [35].

Обоснование использования антибиотиков с высокой внутрипросветной доступностью основано на доказательстве того, что дивертикулы представляют собой мешочки в стенке толстой кишки, которые способствуют захвату кала, бактериальному разрастанию и потенциальному разрушению эпителиальной выстилки, с последующим вовлечением в бактериальную транслокацию слизистой оболочки, что приводит к прогрессирующему воспалению и возникновению осложнений [36]. Эта догма в настоящее время подтверждается фундаментальными исследованиями, указывающими на наличие дисбиоза у пациентов с ДБТК [37]. При этом рифаксимин демонстрирует обширную доказательную эффективность в лечении бактериального разрастания и в тонкой кишке и связанных с ДБТК органических и функциональных желудочно-кишечных расстройств [38, 39].
Незамедлительное хирургическое вмешательство проводится при наличии свободной перфорации, сопровождающейся перитонитом, непроходимости кишечника, при невозможности дренировать абсцесс, кровотечении, ухудшении состояния пациента при проведении консервативной терапии в полном объеме.

Плановое хирургическое лечение является наиболее частым. Операция проводится после соответствующей подготовки кишечника.

Плановое хирургическое вмешательство показано при дивертикулите, при котором отмечается вытекание контрастного материала при инструментальном обследовании; симптомах непроходимости и отсутствии возможности проведения дифференциальной диагностики с колоректальным раком

Плановая операция чаще всего связана с резекцией сигмовидной кишки. Резекцию рекомендуется проводить после окончания антибактериальной терапии [39].

В заключение необходимо отметить, что последние фармакологические подходы в терапии ДБТК направлены на купирование бактериальной агрессии (плохо всасываемые антибиотики, такие как рифаксимин или пробиотики) или воспаления (производные 5-ASA), что ведет к купированию клинической симптоматики, а также предотвращению осложнений. Не вызывает 
сомнения тот факт, что в будущем тактика ведения больных с заболеваними желудочно-кишечного тракта, в том числе ДБТК, будет базироваться на так называемой «микробиот-направленной терапии».

Профилактика ДБТК связана с изменением характера питания. Так, риск манифестации ДБТК (относительный риск = 0,63, $95 \%$ Cl 0,44-0,91) уменьшается у индивидуумов, употреблявших в среднем 32 грам- ма клетчатки в день [40]. Доминирование дивертикулеза отмечается у лиц с индексом массы тела $\geq 30 \mathrm{kг} / \mathrm{M}^{2}$, сидячим образом жизни, при ежедневном употребление продуктов с содержанием растительной клетчатки в дозе $\leq 15$ г/сут, а также при генетически-детерминированных заболеваниях соединительной ткани (синдром Марфана, синдром Элерса-Данлоса, поликистоз почек) [41].

\section{Литература / References}

1 Peery A.F., Crockett S.D., Murphy C.C. et al. Burden and cost of gastrointestinal, liver, and pancreatic diseases in the United States: update 2018. Gastroenterology. 2019; 156:254-272.e11.

2 Osadchuk M.A., Svistunov A.A., Zolotovitskaya A.M. and others. Diverticular disease of the large intestine and its association with polyps and colorectal cancer: clinical-instrumental and immunomorphological research // North Caucasus Medical Newsletter. - 2020. - № 1. - C. 52-57.

3 Everhart J.E., Ruhl C.E. Burden of digestive diseases in the United States part II: lower gastrointestinal diseases. Gastroenterology. 2009;136:741-754.

4 Michael Camilleri, Robert S. Sandler, Anne F. Peery. Etiopathogenetic Mechanisms in Diverticular Disease of the Colon. Cell Mol Gastroenterol Hepatol. 2020; 9(1): 15-32. Published online 2019 Jul 25. doi: 10.1016/j.jcmgh.2019.07.007 PMCID: PMC6881605

5 Rafferty J, Shellito P, Hyman NH, Buie WD. Practice parameters for sigmoid diverticulitis. Dis Colon Rectum. 2006;49(7):939-44. doi: 10.1007/s10350-006-0578-2.

6 Nguyen GC, Sam J, Anand N. Epidemiological trends and geographic variation in hospital admissions for diverticulitis in the United States. World J Gastroenterol. 2011;17(12):1600-1605.

7 Etzioni DA, Mack TM, Beart RW, Jr, et al. Diverticulitis in the United States: 1998-2005: Changing patterns of disease and treatment. Ann Surg 2009; 249: 210-217.

8 Delvaux M. Diverticular disease of the colon in Europe: Epidemiology, impact on citizen health and prevention. Aliment PharmacolTher 2003; 18: S71-S74.

9 Sandler RS, Everhart JE, Donowitz M, Adams E, Cronin K, Goodman C, Gemmen E, Shah S, Avdic A, Rubin $R$. The burden of selected digestive diseases in the United States. Gastroenterology. 2002;122:1500-1511.

10 Alatise OI, Arigbabu AO, Agbakwuru EA, et al. Spectrum of colonoscopy findings in lle-lfe Nigeria. Niger Postgrad Med J 2012; 19: 219-224.

11 Strate L. L. Diverticulosis and dietary fiber: rethinking the relationship. Gastroenterology 142, 205-207 (2012).

12 Meurs-Szojda M. M., Terhaar sive Droste J. S., Kuik D. J.,Mulder C. J. J., Felt-Bersma R. J. F. Diverticulosis and diverticulitis form no risk for polyps and colorectal neoplasia in 4,241 colonoscopies. Int. J. Colorectal. Dis.2008;23(10):979-984. https://doi.org/10.1007/s00384-008-0510-4

13 Regula J. Diverticular Disease and Colorectal Cancer: Incidental Diagnosis or Real Association? Final Answer. J. Clin. Gastroenterol. 2016;50 Suppl.1:S39-40.https://doi.org/10.1097/MCG.0000000000000643

14 Jansen A. Risk factors for colonic diverticular bleeding: A westernized community-based hospital study. World J Gastroenterol 2009; 15: 457-457

15 Jansen A. Risk factors for colonic diverticular bleeding: A westernized community-based hospital study. World J Gastroenterol 2009; 15: 457-457

16 Strate L.L., Morris A.M. Epidemiology, pathophysiology, and treatment of diverticulitis. Gastroenterology. 2019;156:1282-1298.e1.

17 Daniels L., Philipszoon L.E., Boermeester M.A. A hypothesis: important role for gut microbiota in the etiopathogenesis of diverticular disease. Dis Colon Rectum. 2014;57:539-543.

18 Nguyen L.H., Cao Y., Ma W., Jovani M., Liu P.-H., Huttenhower C., Ludvigsson J.F., Wu K., Giovannucci E., Strate L.L., Chan A.T. Chronic antibiotic use and subsequent development of diverticulitis. Gastroenterology. 2019;156(Suppl 1):S1155. 
19 Walker MM, Harris AK. Pathogenesis of diverticulosis and diverticular disease. Minerva GastroenterolDietol. 2016 Dec 14.

20 Wedel T, Barrenschee M, Lange C, Cossais F, Böttner M. Morphologic Basis for Developing Diverticular Disease, Diverticulitis, and Diverticular Bleeding. Viszeralmedizin. 2015 Apr;31(2):76-82. doi: 10.1159/000381431. Review.

21 Scarpignato C, Barbara G, Lanas A, et al. Management of colonic diverticular disease in the third millennium: Highlights from a symposium held during the United European Gastroenterology Week 2017. Therap Adv Gastroenterol 2018; 11: 1756284818771305-1756284818771305.

22 Tursi A, Elisei W, Giorgetti GM, et al. Inflammatory manifestations at colonoscopy in patients with colonic diverticular disease. AlimentPharmacolTher 2011; 33: 358-365.

23 Diverticular disease of the large intestine / A.A. Svistunov, M.A. Osadchuk, D.V. Bala-shev, M.M. Osadchuk. M.: RAMS Publishing House, 2017. - 52 p.

24 Predictive value of the Diverticular Inflammation and Complication Assessment (DICA) endoscopic classification on the outcome of diverticular disease of the colon: An international study. Antonio Tursi,corresponding author / G. Brandimarte [et al.] // United European Gastroenterol J. 2016 Aug; 4(4): 604-613.

25 Tursi A, Brandimarte G, Di Mario F, et al. Development and validation of an endoscopic classification of diverticular disease of the colon: The DICA classification. DigDis 2015; 33: 68-76.

26 Gallo A1, laniro G, Montalto M, Cammarota G. The Role of Biomarkers in Diverticular Disease. J ClinGastroenterol. 2016 Oct;50 Suppl 1:S26-8. doi: 10.1097/MCG.0000000000000648.

27 Tursi A. New physiopathological and therapeutic approaches to diverticular disease: An update. ExpertOpinPharmacother 2014; 15: 1005-1017.

28 Cuomo R, Barbara G, Andreozzi P, et al. Symptom patterns can distinguish diverticular disease from irritable bowel syndrome. Eur J Clin Invest 2013; 43: 1147-1155.

29 Bevan R, Lee TJ, Nickerson C, et al. Non-neoplastic findings at colonoscopy after positive faecal occult blood testing: Data from the English Bowel Cancer Screening Programme. J Med Screen 2014; 21: 89-94

30 Scarpignato C, Barbara G, Lanas A, et al. Management of colonic diverticular disease in the third millennium: Highlights from a symposium held during the United European Gastroenterology Week 2017. Therap Adv Gastroenterol 2018; 11: 1756284818771305-1756284818771305.

31 Carabotti M, Annibale B. Treatment of diverticular disease: An update on latest evidence and clinical implications. Drugs Context 2018; 7: 212526-212526.

32 Carmelo Scarpignato, Giovanni Barbara, Angel Lanas, Lisa L. Strate. Management of colonic diverticular disease in the third millennium: Highlights from a symposium held during the United European Gastroenterology Week 2017. Therap Adv Gastroenterol. 2018; 11: 1756284818771305. Published online 2018 May 20. doi: $10.1177 / 1756284818771305$

33 Tandon A, Fretwell VL, Nunes QM, et al. Antibiotics versus no antibiotics in the treatment of acute uncomplicated diverticulitis - a systematic review and meta-analysis. Colorectal Dis. Epub ahead of print 11 January 2018. DOI: $10.1111 /$ codi. 14013

34 Gargallo Puyuelo CJ, Sopena F, Lanas Arbeloa A. Colonic diverticular disease. Treatment and prevention. Gastroenterol Hepatol 2015; 38: 590-599.

35 Barbara G, Cremon C, Barbaro MR, et al. Treatment of diverticular disease with aminosalicylates: the evidence. J Clin Gastroenterol 2016; 50(Suppl. 1): S60-S63.

36 Barbara G, Scaioli E, Barbaro MR, et al. Gut microbiota, metabolome and immune signatures in patients with uncomplicated diverticular disease. Gut 2017; 66: 1252-1261.

37 Humes DJ, Spiller RC. Review article: the pathogenesis and management of acute colonic diverticulitis. Aliment Pharmacol Ther 2014; 39: 359-370.

38 Cuomo R, Barbara G, Annibale B. Rifaximin and diverticular disease: position paper of the Italian Society of Gastroenterology (SIGE). Dig Liver Dis 2017; 49: 595-603.

39 Italian consensus conference for colonic diverticulosis and diverticular disease. Rosario Cuomo,corresponding author1 Giovanni Barbara,2 Fabio Pace,3 Vito Annese,4 Gabrio Bassotti,5 Gian Andrea Binda,6 Tino Casetti,7 Antonio Colecchia,2 Davide Festi,2 Roberto Fiocca,8 Andrea Laghi,9 Giovanni Maconi,10 Riccardo Nascimbeni,11 Carmelo Scarpignato,12 Vincenzo Villanacci,13 and Bruno Annibale14. United European Gastroenterol J. 2014 Oct; 2(5): 413-442. doi: 10.1177/2050640614547068 
40 Strate L.L., Liu Y.L., Aldoori W.H., et al. Physical activity decreases diverticular complications. Am JGastroenterol 2009; 104:1221-30.

41 Strate L.L., Liu M.S., Aldoori W.H., et al. Obesity increases the risk of diverticulitis and diverticular bleeding. Gastroenterology 2009; 136(1):115-22.

\section{Авторская справка \\ Лысов Николай \\ Александрович \\ Осадчук Максим \\ Михайлович}

\section{Балашов Дмитрий Владимирович}

доктор медицинских наук, профессор, ректор, Медицинский университет «Реавиз», Самара, Россия

кандидат медицинских наук, доцент кафедры клинической медицины, Московский Медицинский университет «Реавиз», Москва, Россия

заведующий отделом организационно-методической и клинико-экспертной работы, Городская поликлиника № 52 Департамента здравоохранения города Москвы», Москва, Россия

кандидат медицинских наук, Главный врач, Городская поликлиника № 52 Департамента здравоохранения города Москвы», Москва, Россия 